\title{
RECONSTRUCTION FOR COLORINGS ON TREES
}

\author{
NAYANTARA BHATNAGAR*, JUAN VERA ${ }^{\dagger}$, ERIC VIGODA ${ }^{\ddagger}$, AND DROR WEITZ §
}

\begin{abstract}
Consider $k$-colorings of the complete tree of depth $\ell$ and branching factor $\Delta$. If we fix the coloring of the leaves, for what range of $k$ is the root uniformly distributed over all $k$ colors (in the limit $\ell \rightarrow \infty$ )? This corresponds to the threshold for uniqueness of the infinite-volume Gibbs measure. It is straightforward to show the existence of colorings of the leaves which "freeze" the entire tree when $k \leq \Delta+1$. For $k \geq \Delta+2$, Jonasson proved the root is "unbiased" for any fixed coloring of the leaves and thus the Gibbs measure is unique. What happens for a typical coloring of the leaves? When the leaves have a non-vanishing influence on the root in expectation, over random colorings of the leaves, reconstruction is said to hold. Non-reconstruction is equivalent to extremality of the free-boundary Gibbs measure. When $k<\Delta / \ln \Delta$, it is straightforward to show that reconstruction is possible (and hence the measure is not extremal).

We prove that for $C>1$ and $k=C \Delta / \ln \Delta$, non-reconstruction holds, i.e., the Gibbs measure is extremal. We prove a strong form of extremality: with high probability over the colorings of the leaves the influence at the root decays exponentially fast with the depth of the tree. Closely related results were also proven recently by Sly. The above strong form of extremality implies that a local Markov chain that updates constant sized blocks has inverse linear entropy constant and hence $O(N \log N)$ mixing time where $N$ is the number of vertices of the tree. Extremality on trees and random graphs has received considerable attention recently since it may have connections to the efficiency of local algorithms.
\end{abstract}

Key words. Reconstruction, Random colorings, Extremality of Gibbs measure

AMS subject classifications. 60J05, 60K35

1. Introduction. Reconstruction on trees arises naturally in a variety of contexts. In evolutionary biology it is intimately related with the efficiency of inferring phylogenetic ancestors [28, 9. It appears in communication theory in the study of noisy computation [12]. Most closely related to the origins of this work, for spin systems from statistical physics, the threshold for reconstruction is equivalent to the threshold for extremality of the infinite-volume Gibbs measure induced by freeboundary conditions (see, e.g., [15), and is closely connected to mixing properties of the single-site update Markov chain known as the Glauber dynamics [4, 24. In fact, Berger et al [4] showed that $O(N \log N)$ mixing time of the Glauber dynamics implies extremality. Recent work of Krzakala et al [20] used statistical physics methods to study extremality for random colorings and random $k$-SAT, and suggests there are interesting connections between thresholds for extremality and efficiency of local algorithms.

Our general setup is a complete tree with branching factor $\Delta$ and depth $\ell$, which we will denote as $T_{\ell}=\left(V_{\ell}, E_{\ell}\right)$. (To clarify, the maximum degree of this tree is $\Delta+1$.) A $k$-coloring is an assignment $\sigma: V_{\ell} \rightarrow[k]=\{1, \ldots, k\}$ where $(v, w) \in E_{\ell}$ implies $\sigma(v) \neq \sigma(w)$. Let $\mu_{\ell}$ denote the uniform measure over $k$-colorings of the tree. Finally, we denote the leaves of the tree $T_{\ell}$ by $L_{\ell}$. When the height of the tree is clear from the context, we will drop the subscript and just use $L$.

In the reconstruction problem, we are asking whether a typical coloring of the leaves influences the conditional measure at the root. In words, we choose a random

\footnotetext{
${ }^{*}$ Department of Statistics, University of California, Berkeley, nayan@stat.berkeley.edu. Research supported in part by NSF grant CCF-0455666 and DOD ONR grant N0014-07-1-05-06.

${ }^{\dagger}$ Department of Management Sciences, University of Waterloo, jvera@uwaterloo.ca

$\ddagger$ College of Computing, Georgia Institute of Technology, vigoda@cc.gatech.edu. Research supported in part by NSF grant CCF-0455666.

$\S \mathrm{Tel}$ Aviv, Israel, dror@drorweitz.com.
} 
coloring of the tree, fix the induced coloring for the leaves, and then choose a random coloring of the internal vertices consistent with the leaves. If the root has some nonvanishing bias to any color then we say reconstruction is possible. Here is a precise definition.

DEFINITION 1.1. The reconstruction problem for $T_{\ell}$ is solvable if there exists $c \in[k]$ such that

$$
\lim _{\ell \rightarrow \infty} \mathbf{E}_{\tau \sim \mu_{\ell}}\left[\mid \mu_{\ell}(\sigma(\text { root })=c \mid \sigma(L)=\tau(L))-1 / k \mid\right]>0 .
$$

Non-reconstruction is equivalent to extremality of the corresponding Gibbs measure of the infinite tree (i.e., it can not be expressed as a convex combination of other Gibbs measures), see, e.g., [15.

In the corresponding reconstruction problem for the Ising model, the reconstruction threshold has been precisely established [6. Recent works have improved bounds on the reconstruction threshold for the case of the hard-core model on weighted independent sets [8, 22, 24. More recently, Borgs et al [5] showed that for binary asymmetric channels, the Kesten-Stigum eigenvalue bound is tight for reconstruction non-solvability in the case of limited asymmetry.

The problem of determining the reconstruction threshold for colorings on the tree was discussed in [7, 25, 29. The threshold for uniqueness of the Gibbs measure on trees is now well-known. In contrast to extremality, uniqueness of the Gibbs measure requires that for every fixed coloring of the leaves, the root is not biased to any color. Jonasson [19] proved that for $k \geq \Delta+2$ the Gibbs measure is unique [19], whereas for $k=\Delta+1$ there are "frozen" boundary conditions in the sense that the root has only one possible color, see [7] for more on the topic of uniqueness for colorings.

It was known [30] that if $k<(1-\varepsilon) \Delta / \ln \Delta$ for any $\varepsilon>0$ then reconstruction is solvable, and more precisely as established in 33, reconstruction is solvable for $\Delta \geq k(\ln k+\ln \ln k+1+o(1))$. Recent works [20, 35] used the so-called replicasymmetry breaking method to conjecture that the reconstruction threshold for $k$ colorings of the tree $T_{\ell}$ is at $\Delta=k(\ln k+\ln \ln k+1+o(1))$. From an algorithmic perspective it is interesting that these bounds on the degree are close to the current best results for efficiently coloring Erdös-Rényi random graphs $G(n, \Delta / n)[1$.

In this work we prove that the reconstruction threshold is at $\frac{\Delta}{\ln \Delta}(1+o(1))$. In particular, we show that for $C>1$ and $k>C \Delta / \ln \Delta$ reconstruction is not possible, with the bias of an average boundary condition decaying exponentially fast with the height of the tree.

TheOREM 1.2. Let $C>1$ and $\varepsilon=\min \{C-1,1 / 3\}$. There are constants $\alpha(\varepsilon)>0, \Delta_{0}=\Delta_{0}(\varepsilon)$, and $\ell_{0}=\ell_{0}(\varepsilon)$ such that for all $\Delta>\Delta_{0}$, every $k>C \Delta / \ln \Delta$, and every color $c \in[k]$, for $\ell>\ell_{0}$,

$$
\mathbf{E}_{\tau \sim \mu_{\ell}}\left[\mid \mu_{\ell}(\sigma(\text { root })=c \mid \sigma(L)=\tau(L))-1 / k \mid\right] \leq \Delta^{-\alpha \ell} .
$$

We also prove a high concentration result, where a much stronger tail bound (on the fraction of biasing boundary conditions) is established.

Theorem 1.3. Let $C>1$ and $\varepsilon=\min \{C-1,1 / 3\}$. There are constants $\alpha(\varepsilon), \alpha^{\prime}(\varepsilon)>0, \Delta_{0}=\Delta_{0}(\varepsilon)$ and $\ell_{0}=\ell_{0}(\varepsilon)$ such that for all $\Delta>\Delta_{0}$, every $k>$ $C \Delta / \ln \Delta$, and every color $c \in[k]$, for $\ell>\ell_{0}$,

$$
\operatorname{Pr}_{\tau \sim \mu_{\ell}}\left[\mid \mu_{\ell}(\sigma(\text { root })=c \mid \sigma(L)=\tau(L))-1 / k \mid>\Delta^{-\alpha \ell}\right] \leq e^{-\Delta^{\alpha^{\prime} \ell}} .
$$


Remark 1.4 (A Note on Comparison with Previous Work). An earlier version of this paper proved the above results for $C>2$, which was the first work giving the right order for the threshold for non-reconstruction [3]. Stated in terms to make comparison with other work easier, it was shown there that non-reconstruction holds when $\Delta \leq$ $\frac{1}{2} k \ln k-o(k \ln k)$. The bound was sharpened by Sly in [34], using independent methods, and showing that non-reconstruction holds when $\Delta \leq k[\ln k+\ln \ln k+1-\ln 2-o(1)]$. Subsequent to this the authors of this paper refined the approach of [3] to obtain the current results, showing non-reconstruction when $\Delta \leq k \ln k-o(k \ln k)$. Though the bound on the reconstruction threshold in [34] is more precise, we feel our results are still of independent interest for the following reasons.

The techniques used in the current work (an extension of those appearing in [3], utilizing ideas from [23]) are independent and different from the analytical approach of [34]. There may be some insight to be gained from this proof approach. Secondly, as a consequence of the strong concentration result stated above, using results of Martinelli et al [23, 24], it follows that a local Markov chain (in each step the colors of a constant number of vertices are updated) has inverse linear entropy constant and hence $O(N \log N)$ mixing time.

We state the relevant definitions before formally stating the result for the dynamics. We consider dynamics on the tree $T_{n}$, and analyze its performance as a function of the volume of the tree $N=\left|T_{n}\right|$. In each step the dynamics updates the colors of a block of vertices. Specifically, for a fixed parameter $\ell$, for every $v \in T_{n}$ let $B_{v, \ell}$ be the subtree (of $T_{n}$ ) of depth $\ell$ rooted at $v$, i.e., $B_{v, \ell}$ includes all vertices in $T_{n}$ that are descendants of $v$ (including $v$ ) and whose distance from $v$ is at most $\ell$. We analyze the dynamics $\mathcal{M}_{\ell}$, which makes heat-bath updates in a random block $B_{v, \ell}$. Specifically, let the state space $\Omega$ be the set of proper $k$-colorings of $T_{n}$. From $X_{t} \in \Omega$, the transition $X_{t} \rightarrow X_{t+1}$ is defined as follows:

- Choose a vertex $v$ uniformly at random from $T_{n}$.

- For all $w \notin B_{v, \ell}$, set $X_{t+1}(w)=X_{t}(w)$.

- Choose $X_{t+1}\left(B_{v, \ell}\right)$ uniformly at random from the set of $k$-colorings consistent with $X_{t+1}\left(T_{n} \backslash B_{v, \ell}\right)$.

The case when $\ell=0$ is the well-studied single-site dynamics known as the Glauber dynamics. It is easy to see that for $k \geq 4$ and for all $\ell, \mathcal{M}_{\ell}$ is an ergodic Markov chain with unique stationary distribution $\pi$ uniformly distributed over $\Omega$. The central question is the mixing time defined as:

$$
T_{\text {mix }}=\max _{X_{0}} \min \left\{t:\left\|P^{t}\left(X_{0}, \cdot\right)-\pi\right\| \leq 1 / 2 e\right\}
$$

where $P^{t}\left(X_{0}, \cdot\right)$ is the distribution after $t$ steps of the dynamics starting from coloring $X_{0}$ and $\|\cdot\|$ denotes variation distance. The choice of the constant $1 / 2 e$ implies that variation distance $\leq \varepsilon$ can be achieved after $\leq\lceil\ln 1 / \varepsilon\rceil T_{\text {mix }}$ steps [2].

We bound the mixing time via the entropy constant. Let $f: \Omega \rightarrow \mathbb{R}$ be an arbitrary test function. The entropy of $f$ is

$$
\operatorname{Ent}(f):=\mathbf{E n t}_{\pi}(f)=\mathbf{E}_{\pi}[f \ln (f)]-\mathbf{E}_{\pi}(f) \ln \left[\mathbf{E}_{\pi}(f)\right],
$$

and the entropy constant of the Markov chain is defined as

$$
\alpha_{\mathrm{E}}=\inf _{f \geq 0} \frac{\operatorname{Ent}(f)-\operatorname{Ent}(P f)}{\operatorname{Ent}(f)}
$$


where the infimum is over non-constant functions $f$. The entropy constant, which bounds the rate of decay of entropy, provides a good bound on the mixing time. In particular, standard results (see, e.g., [10, 13, 27]) imply:

$$
T_{\text {mix }} \leq O\left[\alpha_{\mathrm{E}}^{-1} \log \log \left(\pi_{\text {min }}^{-1}\right)\right] \leq O\left(\alpha_{\mathrm{E}}^{-1} \log N\right) .
$$

We prove the following result about the dynamics.

TheOrem 1.5. Let $C>1$ and $\varepsilon=\min \{C-1,1 / 3\}$. There are constants $\Delta_{0}=\Delta_{0}(\varepsilon)$, and $\ell=\ell(\varepsilon)$ such that for all $\Delta>\Delta_{0}$, every $k>C \Delta / \ln \Delta$, and all $n$, the entropy constant of the dynamics $\mathcal{M}_{\ell}$ on $T_{n}$ satisfies

$$
\alpha_{\mathrm{E}} \geq \frac{1}{4 N}
$$

and consequently the mixing time satisfies

$$
T_{\text {mix }}=O(N \log N) .
$$

It is an interesting open problem to prove $O(N \log N)$ mixing time of the Glauber dynamics for the same range of colors as the above theorem. The best known results for the mixing time of the Glauber dynamics on the tree are Martinelli et al 24] who proved $O(N \log N)$ mixing time when $k \geq \Delta+3$ for arbitrary boundary conditions, and Hayes et al [18] proved polynomial in $N$ mixing time (specifically, $O^{*}\left(N^{4}\right)$ mixing time) when $k>100 \Delta / \log \Delta$ for any planar graph. For complete trees, Goldberg et al [17] and Lucier et al [21] recently showed the mixing time is polynomial for any fixed $k$ and $\Delta$.

Finally, a related problem is the reconstruction threshold on Erdös-Rényi random graphs $G(n, p)$ with $p=\Delta / n$, so-called sparse random graphs. Recent work of Gerschenfeld and Montanari [16] gives sufficient conditions under which extremality on the tree is equivalent to extremality for sparse random graphs. They showed that the conditions are satisfied for the $q$-state Potts model at all temperatures except zero temperature, which is the case of proper colorings. Subsequently in [26, the conditions were also verified in the case of proper colorings.

2. Proof outline and outline of paper. Our proof is divided into two major parts. In the first part, which is contained in Section 3, we prove that with very high probability over the colorings of the leaves, the root is not too highly biased in favor of any color. Roughly, with probability $\geq 1-e^{-\Delta^{\Omega(\ell)}}$ over random colorings $X$ of the leaves, for every color $c$,

$$
\mu_{\ell}(\sigma(\text { root })=c \mid \sigma(L)=X) \leq \Delta^{-\varepsilon / 2}
$$

where $\varepsilon$ is as defined in Theorem 1.3. We prove this statement by analyzing the same recurrences as used by Jonasson [19] in his proof for uniqueness on the tree when $k \geq \Delta+2$. His recurrences express the marginal distribution at the root of a tree of size $\ell$ in terms of the marginals for trees of size $\ell-1$. The difficulty in our setting is that when $k<\Delta+2$ it is unclear if the recurrences converge to the uniform distribution as a fixed point.

In the second part of the proof (which appears in Section 4) we use a two stage coupling similar to that used in 23 together with the bound on the maximum probability of a color from the first part to establish the decay of correlation stated in 
Theorem 1.2. We then add arguments (taken from [23]) to get the stronger Theorem 1.3 .

Finally, in Section 5 we prove Theorem 1.5 establishing fast convergence of the block dynamics for constant sized blocks.

3. Unbiasing leaf colorings. In this section we show that for most colorings of the leaves $X$, at all vertices far enough from the leaves, the color conditioned on $X$ has "sufficient" randomness.

We call a vector $X \in\{\star, 1, \ldots, k\}^{\Delta^{h}}$ a (partial) coloring of the leaves $L_{h}$. The notation $X_{i}=\star$ is used to denote that the $i$-th leaf is not assigned a color. We say that $X$ is allowed if there exists a coloring $\sigma$ of the tree $T_{h}$ which is consistent with $X$, i.e., for every leaf $z$, if $X(z) \in\{1, \ldots, k\}$ then $\sigma(z)=X(z)$.

Given an allowed coloring $X$ of $L_{h}$ and $c \in[k]$ we define $P_{h}(X, c)$ inductively by

$$
P_{0}(X, c)= \begin{cases}1 & \text { if } X=c \\ 1 / k & \text { if } X=\star \\ 0 & \text { oherwise }\end{cases}
$$

$$
P_{h}(X, c)=\frac{\prod_{i=1}^{\Delta}\left(1-P_{h-1}\left(X_{i}, c\right)\right)}{\sum_{d \in[k]} \prod_{i=1}^{\Delta}\left(1-P_{h-1}\left(X_{i}, d\right)\right)}
$$

where $X=\left(X_{1}, \ldots, X_{\Delta}\right)$ with each $X_{i} \in\{\star, 1, \ldots, k\}^{\Delta^{h-1}}$. An inductive argument shows that $P_{h}(X, c)$ is well-defined for all allowed colorings $X$ and all colors $c$.

A simple computation shows that for allowed colorings $X, P_{h}(X, c)$ is, in fact, the probability the root is colored $c$ conditioned on $X$ at the leaves. Note that conditioning on a partial coloring $X$ simply means conditioning on the vertices which are assigned colors in $X$.

LEMMA 3.1. For all $h$, and all allowed colorings $X$ of $L_{h}$, and all $c$,

$$
\mu_{h}\left(\sigma(\text { root })=c \mid \sigma\left(L_{h}\right)=X\right)=P_{h}(X, c)
$$

Proof. The proof is by counting the appropriate sets of colorings and induction on $h$. Let $\Omega_{h}(X, c)$ be the number of colorings of a tree of height $h$ where the root is colored $c$ and the coloring is consistent with $X$. Then,

$$
\mu_{h}\left(\sigma(\text { root })=c \mid \sigma\left(L_{h}\right)=X\right)=\frac{\Omega_{h}(X, c)}{\sum_{d \in k} \Omega_{h}(X, d)}=\frac{\prod_{i=1}^{\Delta} \sum_{f \neq c} \Omega_{h-1}\left(X_{i}, f\right)}{\sum_{d \in k} \prod_{i=1}^{\Delta} \sum_{f \neq d} \Omega_{h-1}\left(X_{i}, f\right)}
$$

By the assumption that $X$ extends to a coloring of $T_{h}$, it must be that each $X_{i}$ extends to a coloring of the tree $T_{h-1}$ hence, $\prod_{i} \sum_{f} \Omega_{h-1}\left(X_{i}, f\right) \neq 0$. Dividing the numerator and denominator by this factor, we obtain

$$
\frac{\prod_{i=1}^{\Delta} 1-\frac{\Omega_{h-1}\left(X_{i}, c\right)}{\sum_{f} \Omega_{h-1}\left(X_{i}, f\right)}}{\sum_{d \in k} \prod_{i=1}^{\Delta} 1-\frac{\Omega_{h-1}\left(X_{i}, d\right)}{\sum_{f} \Omega_{h-1}\left(X_{i}, f\right)}}=\frac{\prod_{i=1}^{\Delta} 1-P_{h-1}\left(X_{i}, c\right)}{\sum_{d \in k} \prod_{i=1}^{\Delta} 1-P_{h-1}\left(X_{i}, d\right)}=P_{h}(X, c)
$$


Henceforth, we assume that $C>1$ and $k=C \Delta / \ln \Delta$. Define the parameter $\varepsilon=\varepsilon(C)=\min \{C-1,1 / 3\}$. Notice that $k>(1+\varepsilon) \Delta / \ln \Delta$. We also assume $\Delta_{0}(\varepsilon)$ is a large constant depending only on $\varepsilon$.

We now give a recursive definition of a coloring of the leaves being unbiasing. In the base case, let $X \in\{\star, 1, \ldots, k\}^{\Delta}$ be a (partial) coloring of $L_{1}$. We say that $X$ is unbiasing if and only if at least $\Delta^{\varepsilon / 2}$ colors do not appear in $X$. For $\ell>1$ let $X=\left(X_{1}, \ldots, X_{\Delta}\right)$ be a coloring of $L_{\ell}$ where $X_{i}$ is the coloring of the leaves of the subtree rooted at the $i$-th child of the root. We say that $X$ is unbiasing if and only if at most $\Delta^{1-\varepsilon}$ of the $X_{i}$ are not unbiasing.

Indeed, given an unbiasing coloring of the leaves, the color at root cannot be too biased.

Lemma 3.2. For any $\ell \geq 1$, any unbiasing coloring $X$ of $L_{\ell}$, and any color $c$,

$$
P_{\ell}(X, c) \leq \Delta^{-\varepsilon / 2} .
$$

The main result in this section is that if $X$ is a random coloring of the leaves $L_{\ell}$, then $X$ is unbiasing with very high probability. We use $X \sim \mu_{\ell}(\sigma(L))$ to denote that $X$ is a random coloring of the leaves of the tree $T_{\ell}$, i.e., choose $\sigma$ from $\mu_{\ell}$ and let $X=\sigma(L)$.

THEOREM 3.3. For all $\ell \geq 1$,

$$
\operatorname{Pr}_{X \sim \mu_{\ell}(\sigma(L))}[X \text { is unbiasing }] \geq 1-e^{-\Delta^{\frac{\ell-(1-\varepsilon)}{2}}} .
$$

For a given $\ell$, let $h(v)$ be the distance (or height) of the vertex $v$ from the leaves $L_{\ell}$, and $X_{v}$ be the restriction of the coloring $X$ to those leaves that are in the subtree rooted at $v$. We call $X$ highly unbiasing if for every $v$ with $h(v) \geq \varepsilon \ell, X_{v}$ is unbiasing.

Corollary 3.4. For all $\ell>\ell_{0}(\epsilon)$,

$$
\operatorname{Pr}_{X \sim \mu_{\ell}(\sigma(L))}[X \text { is highly unbiasing }] \geq 1-e^{-\Delta^{\varepsilon \ell / 3}} .
$$

Proof. This follows from Theorem 3.3 using a simple union bound once we notice that there are $O\left(\Delta^{(1-\varepsilon) \ell}\right)$ vertices $v$ with $h(v) \geq \varepsilon \ell$.

The rest of this section is dedicated to proving Lemma 3.2 and Theorem 3.3 ,

3.1. Properties of partial colorings of the leaves. We now show that an unbiasing coloring of the leaves indeed implies a not-too-biased color at the root.

Proof. [Proof of Lemma 3.2 The proof is by induction on $\ell$. Let $X$ be an unbiasing coloring of the leaves $L_{\ell}$. We need to show that $P_{\ell}(X, c) \leq \Delta^{-\varepsilon / 2}$ for every color $c \in[k]$. For the base case when $\ell=1$, by definition, there are at least $\Delta^{\varepsilon / 2}$ colors not appearing in $X$, and hence $P_{1}(X, c) \leq \Delta^{-\varepsilon / 2}$ for every color $c$. For $\ell>1$, write $X=\left(X_{1}, \ldots, X_{\Delta}\right)$, where $X_{i}$ is the coloring of the leaves of the subtree rooted at the $i$-th child of the root. By definition, at most $\Delta^{1-\varepsilon}$ of the $X_{i}$ are not unbiasing. By induction, if $X_{i}$ is unbiasing then for all colors $c, P_{\ell-1}\left(X_{i}, c\right) \leq \Delta^{-\varepsilon / 2}$. If, however, $X_{i}$ is not unbiasing then there are at most $\Delta^{\varepsilon / 2}$ colors $c$ for which $P_{\ell-1}\left(X_{i}, c\right) \geq \Delta^{-\varepsilon / 2}$ because by Lemma 3.1 we have $\sum_{d \in[k]} P_{h-1}\left(X_{i}, d\right)=1$. Therefore, there is a set $G \subseteq[k]$ of size at least $k-\Delta^{1-\varepsilon} \Delta^{\varepsilon / 2}=k-\Delta^{1-\varepsilon / 2}$ such that for all $d \in G$, and for 
every $i \in[\Delta], P_{\ell-1}\left(X_{i}, d\right) \leq \Delta^{-\varepsilon / 2}$. Hence, we have

$$
P_{\ell}(X, c)=\frac{\prod_{i=1}^{\Delta}\left(1-P_{\ell-1}\left(X_{i}, c\right)\right)}{\sum_{d \in[k]} \prod_{i=1}^{\Delta}\left(1-P_{\ell-1}\left(X_{i}, d\right)\right)} \leq \frac{1}{\sum_{d \in G} \prod_{i=1}^{\Delta}\left(1-P_{\ell-1}\left(X_{i}, d\right)\right)} .
$$

Using the arithmetic-geometric mean inequality,

$$
P_{\ell}(X, c) \leq \frac{1}{|G| \prod_{d \in G} \prod_{i=1}^{\Delta}\left(1-P_{\ell-1}\left(X_{i}, d\right)\right)^{1 /|G|}} \leq \frac{1}{|G|} \exp \left(\frac{1+\Delta^{-\varepsilon / 2}}{|G|} \sum_{d \in G} \sum_{i=1}^{\Delta} P_{\ell-1}\left(X_{i}, d\right)\right)
$$

where the second inequality uses the fact that if $x<\delta<1 / 10$ then $1-x \geq \exp (-(1+$ $\delta) x$ ), and that $\Delta>\Delta_{0}(\varepsilon)$. Using induction and plugging in the lower bound $|G| \geq$ $k-\Delta^{1-\varepsilon / 2} \geq\left(1-\Delta^{-\varepsilon / 3}\right) k$ (for $\Delta$ large enough), we obtain

$$
P_{\ell}(X, c) \leq \frac{1}{|G|} \exp \left(\frac{\left(1+\Delta^{-\varepsilon / 2}\right) \Delta}{|G|}\right) \leq \frac{\Delta^{\frac{1+\Delta^{-\varepsilon / 2}}{(1+\varepsilon)\left(1-\Delta^{-\varepsilon / 3}\right)}}}{k\left(1-\Delta^{-\varepsilon / 3}\right)} \leq \Delta^{-\varepsilon / 2}
$$

Before we go on to bound the probability that a random coloring of the leaves is unbiasing (i.e., prove Theorem 3.3), we state some basic lemmas which give some independence, allowing a recursive solution.

Lemma 3.5. Let $X \sim \mu_{\ell}(\sigma(L))$. For any $0 \leq h \leq \ell$, let $w_{1}, \ldots, w_{\Delta^{\ell-h}}$ denote the vertices which are at depth $\ell-h$ from the root. Let $X=\left(X_{w_{1}}, \ldots, X_{w_{\Delta_{\ell-h}}}\right)$ where $X_{w_{i}}$ is the coloring of the leaves of the subtree rooted at $w_{i}$. Then the $X_{w_{i}}^{\Delta_{i}}$ are identically distributed as $\mu_{h}\left(\sigma\left(L_{h}\right)\right)$.

Proof. Consider the following recursive method for constructing a random coloring of the leaves: choose the color of the root uniformly at random from $k$ colors and independently choose the color of each child from the $k-1$ remaining colors. Now consider a vertex $v$ at height $h$ from the leaves. Since each color is equally likely to appear at $v$, the distribution of the coloring at the leaves of the subtree rooted at $v$ is identical to the distribution over colorings of leaves in a random coloring of $T_{h}$.

Lemma 3.6. Let $X=\left(X_{1}, \cdots, X_{\Delta}\right) \sim \mu_{\ell}(\sigma(L))$ where $X_{i} \in[k]^{\Delta^{\ell-1}}$ is $X$ restricted to the subtree rooted at the $i$-th child of the root. Let $\mathcal{U}_{i}$ denote the event that $X_{i}$ is unbiasing. The events $\mathcal{U}_{i}, 1 \leq i \leq \Delta$ are independent.

Proof. The proof follows from the Markovian property of the Gibbs measure (the configurations on the subtrees are independent of each other once we condition on a spin at the root), and the fact that the event of being unbiasing is symmetric with respect to colors. Formally, for $I \subseteq[\Delta]$, let $\mathcal{U}_{I}=\bigcap_{i \in I} \mathcal{U}_{i}$. It is enough to show that 
if $j \notin I$, then $\mu_{\ell}\left(\mathcal{U}_{j} \mid \mathcal{U}_{I}\right)=\mu_{\ell}\left(\mathcal{U}_{j}\right)$. Now,

$$
\begin{aligned}
\mu_{\ell}\left(\mathcal{U}_{j} \mid \mathcal{U}_{I}\right) & =\sum_{c \in[k]} \mu_{\ell}\left(\sigma(\text { root })=c \mid \mathcal{U}_{I}\right) \cdot \mu_{\ell}\left(\mathcal{U}_{j} \mid \sigma(\text { root })=c, \mathcal{U}_{I}\right) \\
& =\sum_{c \in[k]} \mu_{\ell}\left(\sigma(\text { root })=c \mid \mathcal{U}_{I}\right) \cdot \mu_{\ell}\left(\mathcal{U}_{j} \mid \sigma(\text { root })=c\right) \\
& =\sum_{c \in[k]} \mu_{\ell}(\sigma(\text { root })=c) \cdot \mu_{\ell}\left(\mathcal{U}_{j} \mid \sigma(\text { root })=c\right) \\
& =\mu_{\ell}\left(\mathcal{U}_{j}\right)
\end{aligned}
$$

where the second equality follows from the Markovian property of the Gibbs measure and the third equality uses the fact that the event $\mathcal{U}_{I}$ is symmetric with respect to the color at the root.

We are now ready to complete the proof of the main theorem in this section.

Proof. [Proof of Theorem 3.3 Let $q_{\ell}=\mathbf{P r}_{X \sim \mu_{\ell}(\sigma(L))}[X$ is not unbiasing]. We need to show that

$$
q_{\ell} \leq e^{-\Delta^{(\ell-(1-\varepsilon)) / 2}}
$$

which we will do by induction on $\ell$. For $\ell>1$ let the random coloring $X=$ $\left(X_{1}, \ldots, X_{\Delta}\right)$, where $X_{i}$ is the random coloring of the leaves of the subtree rooted at the $i$-th child of the root. Assuming $\Delta \geq \Delta_{0}(\varepsilon)$ (so that $e^{-\Delta^{\varepsilon / 2}}<\Delta^{-2}$ ) we have

$$
\begin{array}{rrr}
q_{\ell} & =\operatorname{Pr}_{X \sim \mu_{\ell}(\sigma(L))}\left[>\Delta^{1-\varepsilon} \text { of the } X_{i}\right. \text { are not unbiasing] } & \text { (by definition) } \\
& \leq \Delta^{\Delta^{1-\varepsilon}} \cdot\left(q_{\ell-1}\right)^{\Delta^{1-\varepsilon}} & \text { (by Lemmas }[3.6 \text { and } 3.5 \text { ) } \\
& \leq\left(\Delta e^{\left.-\Delta^{(\ell-(2-\varepsilon)) / 2}\right)^{\Delta^{1-\varepsilon}}}\right. & \text { (by induction) } \\
& \leq e^{-\Delta^{(\ell-(1-\varepsilon)) / 2}} . &
\end{array}
$$

In the base case of $\ell=1$ the colors at the leaves are chosen independently (from $k-1$ colors, say from $\{1, \cdots, k-1\}$ ) once the color at the root is fixed. We have to upper bound the probability that there are less than $\Delta^{\varepsilon / 2}$ unused colors. Let $S$ denote the set of unused colors. Let $x_{i, c}$ be a $0-1$ valued random variable which is 1 if the color $c$ is chosen at the vertex $i$. Then the number of unused colors is given by

$$
|S|=\sum_{c=1}^{k-1} \prod_{i=1}^{\Delta}\left(1-x_{i, c}\right)
$$

By linearity of expectation and independence over the children, we have

$$
\mathbf{E}[|S|]=\sum_{c=1}^{k-1} \prod_{i=1}^{\Delta}\left(1-\mathbf{E}\left[x_{i, c}\right]\right)=k\left(1-\frac{1}{k-1}\right)^{\Delta} \geq 10 \Delta^{\varepsilon / 2},
$$

where the last inequality follows by using the fact that for $\Delta>\Delta_{0}(\varepsilon), \Delta \leq(1-$ $\left.\frac{2 \varepsilon}{3}\right)(k-1) \ln (k-1)$ and, for $x<\delta, 1-x \geq \exp (-(1+\delta) x)$. It is not difficult to verify (for example by induction) that for $Y_{c}:=\prod_{i}\left(1-x_{i, c}\right)$, the variables $\left\{e^{t Y_{c}}\right\}_{c \in[q]}$ have negative covariance for any $t>0$. Hence, we can apply the usual Chernoff bounds, (see, e.g., the proof of Proposition 7 of [11]), to show that $\operatorname{Pr}\left[|S|<\Delta^{\varepsilon / 2}\right]=q_{1}<$ $e^{-\Delta^{\varepsilon / 2}}$. 
4. Convergence. In this section we complete the proof of Theorem 1.2 , i.e., establish exponential decay of correlations for the measure. We do that using coupling arguments where disagreements percolate down and up the tree, along the lines suggested in [23. The main addition in our arguments is using the properties of the measure established in the previous section (in particular, Corollary 3.4) to get a better bound on the probability that a disagreement percolates upwards. (In 23] worst boundary conditions were assumed for the upward coupling.)

We will establish a slightly different, but still equivalent to that stated in Theorem 1.2. form of the decay of correlations. For every color $c$, let $\mu_{c, \ell}^{\downarrow}$ be the distribution of the coloring of $L_{\ell}$ conditioned on the color at the root being $c$. For every coloring $X$ of the leaves $L_{\ell}$ let $\mu_{X, \ell}^{\uparrow}$ be the distribution of colors at the root conditioned on $X$ being the coloring of the leaves. Finally, let $\mu_{c, \ell}^{\downarrow \uparrow}$ be the distribution of colors at the root resulting from first choosing $X$ from $\mu_{c, \ell}^{\downarrow}$ and then choosing a color for the root from $\mu_{X, \ell}^{\uparrow}$. Non-reconstruction is equivalent to the dependence of $\mu_{c, \ell}^{\downarrow \uparrow}$ on $c$ vanishing with $\ell$. We establish the decay of this dependence in the following theorem.

THEOREM 4.1. Let $C>1$. There is a constant $\alpha(\varepsilon)>0$ such that for all $\Delta>\Delta_{0}$, every $k>C \Delta / \ln \Delta$, and every pair of colors $c_{1}, c_{2} \in[k]$, for $\ell>\ell_{0}$,

$$
d_{T V}\left[\mu_{c_{1}, \ell}^{\downarrow \uparrow}, \mu_{c_{2}, \ell}^{\downarrow \uparrow}\right] \leq \Delta^{-\alpha \ell}
$$

Indeed, it is not too difficult to see that this theorem implies Theorem 1.2, (See Section [6 for a proof of the equivalence between the two forms of decay of correlations.)

We prove Theorem 4.1 by coupling the two distributions. The coupling consists of two steps, the first is coupling the colorings of the leaves (downward coupling) conditioned on the disagreement at the root, and the second is coupling the color at the root (upward coupling) based on the pair of colorings of the leaves achieved in the first step.

We start by bounding the average Hamming distance between the two coupled colorings of the leaves resulting from the first step. For two colorings $X, Y$, let $H_{\ell}(X, Y)$ stand for the Hamming distance between $X\left(L_{\ell}\right)$ and $Y\left(L_{\ell}\right)$, i.e., the number of leaves of the tree of depth $\ell$ in which $X$ and $Y$ differ.

Lemma 4.2. For every $\ell$ and any $c_{1}, c_{2} \in[k]$ there is a coupling $\nu=\nu_{c_{1}, c_{2}, \ell}^{\downarrow}$ of $\mu_{c_{1}, \ell}^{\downarrow}$ and $\mu_{c_{2}, \ell}^{\downarrow}$ such that

$$
\mathbf{E}_{(X, Y) \sim \nu}\left[H_{\ell}(X, Y)\right]=\left(\frac{\Delta}{k-1}\right)^{\ell} \leq \ln ^{\ell}(\Delta)
$$

Proof. We let $\nu_{c_{1}, c_{2}, \ell}^{\downarrow}$ be the following recursive coupling: For $\ell \geq 1$, starting from the disagreement $\left(c_{1}, c_{2}\right)$ at the root, couple the spins at each of the children of the root independently according to the optimal coupling for each child. For each child vertex, if the resulting spins at this vertex agree then the whole subtree can be coupled to agree. If the spins disagree, say a $\left(c_{1}^{\prime}, c_{2}^{\prime}\right)$ disagreement, continue recursively using the coupling $\nu_{c_{1}^{\prime}, c_{2}^{\prime}, \ell-1}^{\downarrow}$, where for the base case the coupling $\nu_{c_{1}, c_{2}, 0}^{\downarrow}$ is trivial. Notice that in our setting, the probability of a disagreement percolating to a given child is $\kappa=1 /(k-1)$. To see this notice that the distribution at the child conditioned on the color of the root being $c$ is uniform over $[k] \backslash\{c\}$. Thus, conditioned on a $\left(c_{1}, c_{2}\right)$-disagreement at the root, the two distributions at the child can be coupled such that the colors agree with probability $\frac{k-2}{k-1}$, i.e., that they agree whenever a color 
other than $c_{1}, c_{2}$ is chosen. Clearly, the average Hamming distance at the leaves is $\kappa^{\ell} \Delta^{\ell}=\left(\frac{\Delta}{k-1}\right)^{\ell}$. ㅁ

The next ingredient we need is a bound on the distance between two conditional distributions at the root in terms of the Hamming distance between the two boundary colorings. We establish such a bound for boundary colorings that are highly unbiasing. We say that a pair $(X, Y)$ of colorings of the leaves is good if both $(X, Y)$ are highly unbiasing.

LEMMA 4.3. For any given $\ell$ and any good pair $(X, Y)$ of colorings of the leaves $L_{\ell}$

$$
d_{T V}\left[\mu_{X, \ell}^{\uparrow}, \mu_{Y, \ell}^{\uparrow}\right] \leq 2 H_{\ell}(X, Y)\left(2 \Delta^{-\varepsilon / 2}\right)^{(1-\varepsilon) \ell} .
$$

Let us complete the proof of Theorem 4.1 assuming Lemma 4.3. For any pair $(X, Y)$ of colorings of the leaves $L_{\ell}$ let $\nu_{X, Y, \ell}^{\uparrow}$ be the optimal coupling of $\mu_{X, \ell}^{\uparrow}$ and $\mu_{Y, \ell}^{\uparrow}$, i.e., the probability of disagreement between the pair of colors chosen in this coupling is exactly $d_{T V}\left[\mu_{X, \ell}^{\uparrow}, \mu_{Y, \ell}^{\uparrow}\right]$. We then set the coupling $\nu_{c_{1}, c_{2}, \ell}^{\downarrow \uparrow}$ as follows. First, choose a pair $(X, Y)$ of colorings of the leaves from $\nu_{c_{1}, c_{2}, \ell}^{\downarrow}$; then, choose a pair of colors at the root from the coupling $\nu_{X, Y, \ell}^{\uparrow}$. Notice that $\nu_{c_{1}, c_{2}, \ell}^{\downarrow \uparrow}$ is indeed a coupling of $\mu_{c_{1}, \ell}^{\downarrow \uparrow}$ and $\mu_{c_{2}, \ell}^{\downarrow \uparrow}$. We need to bound the probability that the two colors at the root chosen according to this coupling disagree. Let $\left(c, c^{\prime}\right)$ be the pair of colors chosen from this couplings. We have

$$
\begin{aligned}
\operatorname{Pr}\left[c \neq c^{\prime}\right]= & \sum_{X, Y} \nu_{c_{1}, c_{2}, \ell}^{\downarrow}(X, Y) \cdot d_{T V}\left[\mu_{X, \ell}^{\uparrow}, \mu_{Y, \ell}^{\uparrow}\right] \\
\leq & \sum_{\operatorname{good}(X, Y)} \nu_{c_{1}, c_{2}, \ell}^{\downarrow}(X, Y) \cdot 2 H_{\ell}(X, Y)\left(2 \Delta^{-\varepsilon / 2}\right)^{(1-\varepsilon) \ell} \\
& +\sum_{\operatorname{bad}(X, Y)} \nu_{c_{1}, c_{2}, \ell}^{\downarrow}(X, Y) \cdot 1 \\
\leq & 2\left(2 \Delta^{-\varepsilon / 2}\right)^{(1-\varepsilon) \ell} \sum_{X, Y} \nu_{c_{1}, c_{2}, \ell}^{\downarrow}(X, Y) \cdot H_{\ell}(X, Y) \\
& +2 \operatorname{Pr}_{X \sim \mu_{\ell}}[X \text { is not highly unbiasing }] \\
\leq & 2\left(2 \Delta^{-\varepsilon / 2}\right)^{(1-\varepsilon) \ell} \cdot \ln ^{\ell}(\Delta)+2 e^{-\Delta^{\varepsilon \ell / 3}} \\
\leq & \Delta^{-\varepsilon \ell / 4}
\end{aligned}
$$

assuming $\Delta \geq \Delta_{0}(\varepsilon)$. Notice that in the first inequality above we used Lemma 4.3 , in the second we used a simple union bound together with the fact that the event of having a highly-unbiasing coloring of the leaves is independent of the color at the root, and in the third inequality we applied Lemma 4.2 to bound the first expression and Corollary 3.4 to bound the second. This completes the proof of Theorem 4.1 assuming Lemma 4.3 .

4.1. Proof of Lemma 4.3. The proof of the lemma goes along similar lines to those in [23], i.e., reducing to the case of a single disagreement using a sequence of boundary colorings where one leaf is changed at a time, and then using a coupling argument to bound the probability that the disagreement at the leaf percolates all the way up to the root. However, our argument differs in two points. The first is that here we have to make sure that the intermediate boundary colorings are also 
valid, and more importantly, that they are highly-unbiasing. The second difference is that we use the fact that the colorings are highly unbiasing to get a better bound on the probability of a disagreement percolating upwards. (In our setting, if we do not assume highly unbiasing colorings at the leaves then in the worst case the disagreement may percolate upwards with probability 1.)

Let $(X, Y)$ be a pair of colorings of the leaves, $m=H(X, Y)$, and $\left(d_{1}, \ldots, d_{m}\right)$ be an enumeration of the leaves in which $X$ and $Y$ differ. We construct a sequence of colorings $X=Z_{0}, Z_{1}, \ldots, Z_{m}, \ldots, Z_{2 m}=Y$ going from $X$ to $Y$ by un-conditioning the sites in which $X, Y$ differ one at a time and then adding the condition according to $Y$ one site at a time. Formally, for $0 \leq i \leq m, Z_{i}(v)=\star$ if $v=d_{j}$ for some $1 \leq j \leq i$, and otherwise $Z_{i}(v)=X(v)$. For $i \geq m, Z_{i}(v)=\star$ if $v=d_{j}$ for some $1 \leq j \leq 2 m-i$, and otherwise $Z_{i}(v)=Y$. It is easy to see that indeed for every $i \geq 0,\left(Z_{i}, Z_{i+1}\right)$ differ at exactly one leaf. We now show that each of the $Z_{i}$ is a valid highly-unbiasing coloring.

Claim 4.4. Let $X, X^{\prime}$ be assignments from the leaves $L_{\ell}$ to $\{\star, 1, \ldots, k\}$ such that for all $v \in L_{\ell}$ we have $X^{\prime}(v) \neq \star$ implies $X^{\prime}(v)=X(v)$. If $X$ is a valid unbiasing coloring of $L_{\ell}$ then so is $X^{\prime}$.

Proof. It is clear that if $X$ is a valid coloring then so is $X^{\prime}$ because $X^{\prime}$ can be extended to $X$. The unbiasing implication follows by induction on $\ell$ from the recursive definition of being unbiasing once we establish it for the base case of $\ell=1$. Indeed, for $\ell=1$, the number of unused colors in $X^{\prime}$ is at least the number in $X$. Thus, if $X$ is unbiasing then so is $X^{\prime}$.

Corollary 4.5. Let $X, X^{\prime}$ be assignments from the leaves $L_{\ell}$ to $\{\star, 1, \ldots, k\}$ such that for all $v \in L_{\ell}$ we have $X^{\prime}(v) \neq \star$ implies $X^{\prime}(v)=X(v)$. If $X$ is a valid highly-unbiasing coloring of $L_{\ell}$ then so is $X^{\prime}$.

Now, by the triangle inequality, the proof of Lemma 4.3 will be concluded once we show the following.

Lemma 4.6. Let $Z, Z^{\prime}$ be two highly-unbiasing colorings of $L_{\ell}$ that differ at exactly one vertex $v \in L_{\ell}$. Then,

$$
d_{T V}\left[\mu_{\ell}(\sigma(\text { root }) \mid \sigma(L)=Z), \mu_{\ell}\left(\sigma(\text { root }) \mid \sigma(L)=Z^{\prime}\right)\right] \leq\left(2 \Delta^{-\varepsilon / 2}\right)^{(1-\varepsilon) \ell} .
$$

The proof of the above lemma goes by an upward coupling argument similar to that used in [23, that is, we couple the spins along the path from $v$ to the root using a recursive coupling similar to that used for the downward coupling. Specifically, given the disagreement at $v$, we first couple the colors at the immediate ancestor $u$ (of $v$ ) using an optimal coupling that minimizes the probability of disagreement at $u$. If the resulting spins at $u$ agree, then we couple the rest of the path with total agreement. If the spins at $u$ disagree then we continue recursively. The variation distance at the root is bounded by the probability that the disagreement at the leaf $v$ percolates along the path from $v$ all the way to the root. Thus, the important missing ingredient is a bound on the probability of disagreement at a vertex $u$ given a disagreement at (exactly) one of its children (when the coloring of the leaves is $Z$ ). This can be done once we have a bound on the maximum probability of any color at $u$, as exemplified by the following proposition, which is taken from 24 .

Proposition 4.7. Let $\mu$ be a distribution of proper coloring of some graph according to some boundary condition, and let $\mu_{u}$ be the marginal of $\mu$ at $u$. Consider now the distribution resulting from the same setting when adding a new neighbor to $u$ (connected only to u) and fixing the neighbor's color to $c$. Let $\mu_{u}^{c}$ be the marginal of 
the latter distribution at $u$. Then for any two colors $c_{1}, c_{2}$,

$$
d_{T V}\left[\mu_{u}^{c_{2}}, \mu_{u}^{c_{1}}\right]=\max \left\{\mu_{u}^{c_{2}}\left(c_{1}\right), \mu_{u}^{c_{1}}\left(c_{2}\right)\right\}=\max \left\{\frac{\mu_{u}\left(c_{1}\right)}{1-\mu_{u}\left(c_{2}\right)}, \frac{\mu_{u}\left(c_{2}\right)}{1-\mu_{u}\left(c_{1}\right)}\right\} .
$$

In particular, if we let $p_{u}^{\max }=\max _{c} \mu_{u}(c)$ then $d_{T V}\left[\mu_{u}^{c_{2}}, \mu_{u}^{c_{1}}\right] \leq p_{u}^{\max } /\left(1-p_{u}^{\max }\right)$.

Proof. We first notice that $\mu_{u}^{c}$ is $\mu_{u}$ conditioned on the resulting color not being $c$, i.e., for every color $c^{\prime} \neq c, \mu_{u}^{c}\left(c^{\prime}\right)=\mu_{u}\left(c^{\prime}\right) /\left(1-\mu_{u}(c)\right)$. Assume without loss of generality that $\mu_{u}\left(c_{1}\right) \geq \mu_{u}\left(c_{2}\right)$. It follows that for every color $c^{\prime} \neq c_{1}, \mu_{u}^{c_{2}}\left(c^{\prime}\right) \leq$ $\mu_{u}^{c_{1}}\left(c^{\prime}\right)$. Therefore, $d_{T V}\left[\mu_{u}^{c_{2}}, \mu_{u}^{c_{1}}\right]=\mu_{u}^{c_{2}}\left(c_{1}\right)$. $\square$ Going back to our setting of the upward coupling, Lemma 4.6 will follow once we show that for highly-unbiasing $Z, Z^{\prime}$, for every vertex $w$ on the path from $v$ to the root which is at height $\geq \varepsilon \ell$, given that the disagreement percolated to $w$, the probability that the disagreement percolates to the immediate ancestor $u$ of $w$ is at most $2 \Delta^{-\varepsilon / 2}$. Notice that once a color at $w$ is fixed, the distribution at $u$ is independent of the rest of the coloring of the subtree rooted at $w$, and so that subtree (except for $w$ ) can be completely ignored. Thus, we are in position to use Proposition 4.7 to bound the probability of disagreement at $u$ given a disagreement at $w$ by $p_{u}^{\max } /\left(1-p_{u}^{\max }\right)$, where $p_{u}^{\max }$ in our setting is the maximum probability of a color at $u$ conditioned on $Z$ at the leaves and when the edge $(u, w)$ is removed from $T_{\ell}$ (i.e., the subtree rooted at $w$ is removed from $T_{\ell}$ ).

The fact that $Z$ is highly unbiasing implies that the conditional probability $p_{u}^{\max }$ can be bounded.

Lemma 4.8. Let $u$ be a vertex of the tree $T_{\ell}$ at distance at least $\varepsilon \ell$ from the leaves and $Z$ a highly unbiasing coloring of $L_{\ell}$. Let $p_{u}^{\max }$ be as defined above. Then,

$$
p_{u}^{\max } \leq \Delta^{-\varepsilon / 2} .
$$

Proof. The proof is by the same arguments as in Lemma 3.2 so we don't repeat them, except to point out the following. The probability considered in Lemma 3.2 is that of the subtree rooted at $u$ conditioned on $Z_{u}$, i.e., this subtree is disconnected from the rest of $T_{\ell}$. Here, the distribution considered is when $u$ is still connected to the rest of $T_{\ell}$, but with the subtree rooted at $w$ removed from the tree. However, it is not too difficult to see that if $Z$ is highly unbiasing then the same bound on the maximum probability applies to the distribution considered here.

Consider the orientation of $T_{\ell}$ (after removing the subtree rooted at $w$ ) so it is rooted at $u$. Since $Z$ is a highly-unbiasing boundary coloring of $T_{\ell}$ (in its original orientation) then at least $\Delta-1$ of the children of $u$ are unbiasing (those that are its children in the original orientation), and hence $Z$ is a highly unbiasing boundary condition for the (irregular) newly-oriented tree rooted at $u$. In the newly oriented tree, the distribution considered in Lemma 3.2 does correspond to that considered here for $p_{u}^{\max }$.

By the lemma, for every vertex $u$ at distance at least $\varepsilon \ell$ from the leaves, $p_{u}^{\max } /(1-$ $\left.p_{u}^{\max }\right) \leq 2 \Delta^{-\varepsilon / 2}$ and hence

$$
d_{T V}\left[\mu_{\ell}(\sigma(\text { root }) \mid \sigma(L)=Z), \mu_{\ell}\left(\sigma(\text { root }) \mid \sigma(L)=Z^{\prime}\right)\right] \leq\left(2 \Delta^{-\varepsilon / 2}\right)^{(1-\varepsilon) \ell},
$$

completing the proof of Lemma 4.6 and hence the proof of Lemma 4.3 .

4.2. Concentration. We now go on to prove Theorem 1.3 i.e., establish that not only does a typical boundary coloring yield a near uniform distribution of colors at the root, but that the fraction of boundary colorings having a bias at the root are 
extremely small. The main additional technical tool that we use here is a reduction given in [23] from the tail bound stated in Theorem 1.3 to an appropriate tail bound in the coupling $\nu^{\downarrow}$.

Lemma 4.9. For any given $\ell, \delta>0$ and $A \geq 0$, if for every $c_{1}, c_{2} \in[k]$ there exists a coupling $\nu$ of $\mu_{c_{1}, \ell}^{\downarrow}$ and $\mu_{c_{2}, \ell}^{\downarrow}$ such that

$$
\operatorname{Pr}_{(X, Y) \sim \nu}\left[d_{T V}\left[\mu_{X, \ell}^{\uparrow}, \mu_{Y, \ell}^{\uparrow}\right]>\delta^{3}\right] \leq A
$$

then for every $c \in[k]$,

$$
\operatorname{Pr}_{X \sim \mu_{\ell}}\left[\left|\mu_{X, \ell}^{\uparrow}(c)-1 / k\right|>2 \delta\right] \leq 2\left(e^{-1 / \delta}+\frac{A}{\delta}\right) .
$$

Proof. Arguments proving a similar statement to that of this lemma are given in Section 5.2 of [23. For completeness, we repeat the proof in Section 6 ]

As we show next, the coupling $\nu^{\downarrow}$ constructed above satisfies the hypothesis of Lemma 4.9 with the necessary parameters. In fact, the only ingredient missing in establishing the necessary tail bound for $\nu^{\downarrow}$ is a tail bound on the number of disagreements in the coupling, which in turn follows from the (product) percolation nature of the coupling.

Lemma 4.10. For every $\ell>\ell_{0}(\varepsilon)$ and $c_{1}, c_{2} \in[k]$

$$
\operatorname{Pr}_{(X, Y) \sim \nu_{c_{1}, c_{2}, \ell}^{\downarrow}}\left[H_{\ell}(X, Y)>\Delta^{\varepsilon \ell / 8}\right] \leq e^{-\Delta^{\varepsilon \ell / 10}} .
$$

Proof. Recall from Lemma 4.2 that the average Hamming distance between $X, Y$ is $\leq \ln ^{\ell} \Delta$. The tail bound can then be established as done in, e.g., Lemma 5.5 of [23. For completeness, we give another proof in Section 6, $\mathrm{Q}$

Now, let us refine the notion of a pair of colorings $(X, Y)$ being good by saying that a pair $(X, Y)$ of colorings of the leaves is very good if $(X, Y)$ is good and $H_{\ell}(X, Y) \leq$ $\Delta^{\varepsilon \ell / 8}$. It follows from Lemma 4.3 that for any very-good pair $(X, Y)$,

$$
d_{T V}\left[\mu_{X, \ell}^{\uparrow}, \mu_{Y, \ell}^{\uparrow}\right] \leq \Delta^{-\varepsilon \ell / 8}
$$

On the other hand, by Lemma 4.10 and Corollary 3.4 (where the latter is used as in the proof of Theorem 4.1) we have that

$$
\operatorname{Pr}_{(X, Y) \sim \nu_{c_{1}, c_{2}, \ell}^{\downarrow}}[(X, Y) \text { is not very good }] \leq e^{-\Delta^{\varepsilon \ell / 10}}+2 e^{-\Delta^{\varepsilon \ell / 3}} \leq 2 e^{-\Delta^{\varepsilon \ell / 10}} .
$$

Thus, $\nu_{c_{1}, c_{2}, \ell}^{\downarrow}$ satisfies the hypothesis of Lemma 4.9 with $\delta=\Delta^{-3 \ell}$ and $A=2 e^{-\Delta^{\varepsilon \ell / 10}}$, completing the proof of Theorem 1.3 .

5. Entropy constant for the block dynamics. In this section we prove the rapid mixing result for the block dynamics as stated in Theorem[1.5. i.e., we establish a lower bound on the entropy constant associated with this dynamics when the block height is a large enough constant. We rely on results established in [23, where strong concentration of the measure as established in Theorem 1.3 above is shown to imply the necessary bound on the entropy constant of the block dynamics. We give here the high level motivation behind the kind of argument made in 23 and some necessary adaptations to our setting, while for the main technical result we simply cite the 
relevant theorems from [23. Notice that while the arguments in 23] are written for the Ising model, they hold for general models on trees. (We refer to [31] for the general version of these arguments.)

To see the connection between strong concentration of the measure and the entropy constant, recall that the dynamics makes heat-bath updates in a random block, i.e., erases the local entropy in the updated block. Thus, the amount of entropy erased in one step of the dynamics is the average (over blocks) of local entropies, and in order to bound the entropy constant it is enough to show that the global entropy of every function is not much more than the sum (over blocks) of local entropies of the same function. The ability to express the global entropy of every function as the sum of local entropies up to a constant factor can be thought of as manifestation of locality in the equilibrium measure. Indeed, the main technical result in [23] is that on trees, strong concentration of the measure as in Theorem 1.3 is equivalent to the ability to express entropy locally as above.

To make things formal, let $f$ be a function on the space of colorings $\Omega$. The local entropy of $f$ in a subset $A$ of $T_{n}$ is $\mathbf{E}\left[\operatorname{Ent}\left(f \mid \sigma_{T_{n} \backslash A}\right)\right]$. Consider the block dynamics $\mathcal{M}_{l}$ and let $P_{v}$ be the transition matrix of the dynamics when the block to be updated is the one rooted at $v$. Since the dynamics makes heat-bath updates of the block $B_{v, \ell}$ then for any function $f, P_{v} f(\sigma)=\mathbf{E}\left(f \mid \sigma_{T_{n} \backslash B_{v}, \ell}\right)$, while $P f=\frac{1}{N} \sum_{v \in T_{n}} P_{v} f$. Now, for any subset $A$ of $T_{n}$ we have by definition of entropy that

$$
\operatorname{Ent}(f)=\operatorname{Ent}\left[\mathbf{E}\left(f \mid \sigma_{A}\right)\right]+\mathbf{E}\left[\operatorname{Ent}\left(f \mid \sigma_{A}\right)\right] .
$$

In particular, we have that

$$
\operatorname{Ent}(f)-\operatorname{Ent}\left(P_{v} f\right)=\mathbf{E}\left[\operatorname{Ent}\left(f \mid \sigma_{T_{n} \backslash B_{v, \ell}}\right)\right],
$$

i.e., the effect of applying $P_{v}$ is to erase the local entropy of $f$ in $B_{v, \ell}$. Let us denote the sum of local entropies as

$$
\mathcal{E}_{\ell}:=\sum_{v \in T_{n}} \mathbf{E}\left[\operatorname{Ent}\left(f \mid \sigma_{T_{n} \backslash B_{v, \ell}}\right)\right]
$$

Since entropy is a convex functional then

$$
\operatorname{Ent}(P f)=\operatorname{Ent}\left(\frac{1}{N} \sum_{v \in T_{n}} P_{v} f\right) \leq \frac{1}{N} \sum_{v \in T_{n}} \operatorname{Ent}\left(P_{v} f\right) .
$$

In particular,

$$
\operatorname{Ent}(f)-\operatorname{Ent}(P f) \geq \frac{1}{N} \sum_{v \in T_{n}}\left[\operatorname{Ent}(f)-\operatorname{Ent}\left(P_{v} f\right)\right]=\frac{1}{N} \cdot \mathcal{E}_{\ell}
$$

Thus, in order to prove the lower bound on the entropy constant stated in Theorem 1.5 . it is enough to show that for an appropriate choice of the block height $\ell=\ell(\varepsilon)$ (i.e., not depending on $n$ ),

$$
\inf _{f} \frac{\mathcal{E}_{\ell}(f)}{\operatorname{Ent}(f)} \geq \frac{1}{4}
$$

Now, a key technical ingredient in 23 is that strong concentration of the measure as established in Theorem 1.3 implies (5.1). In order to state the formal implication 
we need a couple of more definitions. For a vertex $v \in T_{n}$, let $T_{v}$ be the subtree rooted at $v$. For a color $c \in[k]$, let $\mu_{T_{v}}^{c}$ be the uniform distribution of colorings of $T_{v}$ conditioned on the parent of $v$ being colored $c$. For $v$ whose distance from the leaves is at least $\ell$, let $L_{v, \ell}$ be the set of vertices at distance $\ell$ below $v$. (Equivalently, $L_{v, \ell}$ is the bottom boundary of $B_{v, \ell}$.) The following theorem combines Theorems 3.4 and 5.3 of 23] (or Theorem 5.10 and Lemma 5.18 in 31, where the formulation is closer to the one here).

ThEOREM 5.1. For some absolute constant $\alpha^{*}$, the following implication is true for every choice of $\ell \geq 1$. If for every vertex $v$ whose distance from the leaves is at least $\ell$ and for any two colors $c_{1}, c_{2}, \mu \equiv \mu_{T_{v}}^{c_{1}}$ satisfies

$$
\operatorname{Pr}_{\tau \sim \mu}\left[\left|\frac{\mu\left(\sigma(v)=c_{2} \mid \sigma\left(L_{v, \ell}\right)=\tau\left(L_{v, \ell}\right)\right)}{\mu\left(\sigma(v)=c_{2}\right)}-1\right|>\frac{1}{\alpha^{*} k^{4}(\ell+1)^{2}}\right] \leq e^{-2 \alpha^{*} k^{4}(\ell+1)^{2}}
$$

then for every function $f \geq 0$, $\operatorname{Ent}(f) \leq 4 \mathcal{E}_{\ell}$. We conclude by noticing that from Theorem 1.3 (see the following Remark 5.2 for a proviso) we get that (5.2) (and therefore (5.1) ) holds for an appropriately chosen $\ell$, e.g., $\ell \geq \frac{10 \alpha^{*}}{\min \left\{\alpha, \alpha^{\prime}\right\}}$ suffices where $\alpha^{*}$ is the absolute constant from Theorem 5.1. and $\alpha=\alpha(\varepsilon)$ and $\alpha^{\prime}=\alpha^{\prime}(\varepsilon)$ are from Theorem 1.3 ,

REMARK 5.2. Notice that Theorem 5.1 requires us to prove the strong concentration of the measure stated in Theorem 1.3 in a slightly more general scenario. Specifically, we need to show similar bounds for a random boundary coloring of $B_{v, \ell}$ conditioned on an arbitrary color at the parent of $v$ and when this boundary coloring is chosen from the measure on $T_{n}$. Notice, however, that the distribution of colorings at the boundary of $B_{v, \ell}$ under the distribution of colorings of $T_{n}$ is exactly the same as the distribution of colorings of the leaves of the smaller tree in which the vertices on the boundary of $B_{v, \ell}$ are the leaves. As for fixing an arbitrary color above $v$, it is straightforward that the concentration stated in Theorem 1.3 holds in this scenario as well. (In this case the distribution at the root is uniform over $k-1$ colors so the statement of the theorem is modified with $\frac{1}{k-1}$ replacing $\frac{1}{k}$.)

REMARK 5.3. In [23] (and [24, 31]), the bound in (5.1) is used to bound the mixing time of the single-site dynamics. This is done by introducing an extra factor that expresses the decomposition of the entropy in a block (under an arbitrary boundary condition) into the local entropy (or variance) coming from single sites in the block. This factor is bounded by the mixing time of the single-site dynamics in the block, which in the setting of [23, 24, 31] is a constant depending on the block size. However, in our setting there are valid boundary colorings of the block for which the single-site dynamics is disconnected (i.e., infinite mixing time) so we cannot apply the same reasoning and suffice with a bound on the mixing time of the block dynamics.

6. Leftover Proofs. Here we provide the remaining proofs. Most of the arguments given in this section already appear in the references mentioned in the main text, but we give them here for completeness and in order for the proofs to correspond exactly to the formulations of the statements made in this paper.

We start by showing that the form of decay of correlations stated in Theorem 4.1] is indeed equivalent to that stated in the main Theorem[1.2. Let $\alpha_{c, \ell}=\mathbf{E}_{X \sim \mu_{\ell}}\left[\mid \mu_{X, \ell}^{\uparrow}(c)-\right.$ $1 / k \mid]$ and $\beta_{c, \ell}=\left|\mu_{c, \ell}^{\downarrow \uparrow}(c)-1 / k\right|$. Notice that Theorem 4.1 establishes that for every $c$ and $\ell \beta_{c, \ell}=\Delta^{-\Omega(\ell)}$, while for Theorem 1.2 we need to show that for every $c$ and $\ell$, $\alpha_{c, \ell}=\Delta^{-\Omega(\ell)}$. The following proposition shows that the two statements are indeed equivalent. 
Proposition 6.1. For every $c$ and $\ell, \frac{\beta_{c, \ell}}{k-1} \leq \alpha_{c, \ell} \leq \sqrt{\beta_{c, \ell}}$.

Proof. Let $g_{c, \ell}(\cdot)$ be the function on coloring of the leaves $L_{\ell}$ defined as follows:

$$
g_{c, \ell}(X)=\frac{\mu_{c, \ell}^{\downarrow}(X)}{\mu_{\ell}(X)}=\frac{\mu_{X, \ell}^{\uparrow}(c)}{\mu_{\ell}\left(\sigma_{\text {root }}=c\right)}=k \cdot \mu_{X, \ell}^{\uparrow}(c) .
$$

Notice that $\mathbf{E}_{X \sim \mu_{\ell}}\left[g_{c}(X)\right]=1$. Furthermore, $\alpha_{c, \ell}=\frac{1}{k} \mathbf{E}_{X \sim \mu_{\ell}}\left[\left|g_{c}(X)-1\right|\right]$, and $\beta_{c, \ell}=$ $\frac{1}{k} \mathbf{E}_{X \sim \mu_{\ell}}\left[\left|g_{c}(X)\right|^{2}-1\right]=\frac{1}{k} \mathbf{E}_{X \sim \mu_{\ell}}\left[\left|g_{c}(X)-1\right|^{2}\right]$. The first inequality of the proposition then follows from the fact that $\left\|g_{c}\right\|_{\infty} \leq k$, while the second inequality follows from Cauchy-Schwartz. $\mathrm{c}$

We now provide the omitted proofs of the two lemmas in Section 4.2 ,

Proof. [Proof of Lemma 4.9] First, notice that since $\mu_{\ell}\left(\sigma\left(L_{\ell}\right)\right)$ is a convex combination of $\mu_{c, \ell}^{\downarrow}$ we can assume w.l.o.g. that for every color $c$ we have a coupling of $\mu_{c, \ell}^{\downarrow}$ and $\mu_{\ell}$ with properties as in the hypothesis of the lemma. Using the same definition of $g_{c, \ell}$ as in Proposition 6.1, for any $\alpha \geq 0$ we then have that

$$
\operatorname{Pr}_{X \sim \mu_{c, \ell}^{\downarrow}}\left[g_{c, \ell}(X) \geq 1+\alpha\right] \leq \operatorname{Pr}_{X \sim \mu_{\ell}}\left[g_{c, \ell}(X) \geq 1+\alpha-k \delta^{3}\right]+A .
$$

On the other hand, by the definition of $g_{c, \ell}$,

$$
\operatorname{Pr}_{X \sim \mu_{c, \ell}^{\downarrow}}\left[g_{c, \ell}(X) \geq 1+\alpha\right] \geq(1+\alpha) \operatorname{Pr}_{X \sim \mu_{\ell}}\left[g_{c, \ell}(X) \geq 1+\alpha\right] .
$$

Combining the above two inequalities we get that

$$
\operatorname{Pr}_{X \sim \mu_{\ell}}\left[g_{c, \ell}(X) \geq 1+\alpha\right] \leq\left(\frac{1}{1+\alpha}\right)\left(\operatorname{Pr}_{X \sim \mu_{\ell}}\left[g_{c, \ell}(X) \geq 1+\alpha-k \delta^{3}\right]+A\right) .
$$

In particular, for every non-negative integer $m$, if we apply the above inequality $m+1$ times, increasing $\alpha$ by $k \delta^{3}$ each time, we get that

$\operatorname{Pr}_{X \sim \mu_{\ell}}\left[g_{c, \ell}(X) \geq 1+\alpha+m k \delta^{3}\right] \leq(1+\alpha)^{-(m+1)}+\frac{A}{k \alpha} \leq e^{-\alpha(m+1) /(1+\alpha)}+\frac{A}{\alpha}$.

Now, by setting $\alpha=k \delta$ and $m=\left\lfloor(1 / \delta)^{2}\right\rfloor$ we get that

$$
\operatorname{Pr}_{X \sim \mu_{\ell}}\left[g_{c, \ell}(X) \geq 1+2 k \delta\right] \leq e^{-\delta(m+1)}+\frac{A}{\delta} .
$$

Since the event $g_{c, \ell}(X) \geq 1+2 k \delta$ is equivalent to $\mu_{X, \ell}^{\uparrow}(c)-1 / k \geq 2 \delta$, this establishes the bound on the positive side of the tail in Lemma 4.9. The negative side of the tail is established along similar lines once we notice that from the hypothesis we have for any $\alpha \geq 0$

$$
\operatorname{Pr}_{X \sim \mu_{\ell}}\left[g_{c, \ell}(X) \leq 1-\alpha-k \delta^{3}\right] \leq \operatorname{Pr}_{X \sim \mu_{c, \ell}^{\downarrow}}\left[g_{c, \ell}(X) \leq 1-\alpha\right]+A
$$

and by definition of $g_{c, \ell}$,

$$
\operatorname{Pr}_{X \sim \mu_{c, \ell}^{\downarrow}}\left[g_{c, \ell}(X) \leq 1-\alpha\right] \leq(1-\alpha) \operatorname{Pr}_{X \sim \mu_{\ell}}\left[g_{c, \ell}(X) \leq 1-\alpha\right]
$$

Proof. [Proof of Lemma 4.10 Recall that the coupling $\nu^{\downarrow}$ is constructed recursively such that if the spin at a vertex $v$ agree then all of its children are coupled with 
agreement with probability 1 , while if there is a disagreement at $v$ then its children are coupled independently where the probability of disagreement in each of the $\Delta$ children is $1 /(k-1)$. Thus, if we let $D_{i}$ be the random variable counting the number of disagreements at level $i$ in this coupling then $D_{0}=1$ and $D_{i+1}$ is distributed as $\operatorname{Bin}\left(\Delta D_{i}, \frac{1}{k-1}\right)$. We need to establish an upper bound on $\operatorname{Pr}\left(D_{\ell} \geq \Delta^{\varepsilon \ell / 8}\right)$. Let $i_{0}=\varepsilon \ell / 9$. For every $i_{0} \leq i \leq \ell$ we say that a failure occurred at level $i$ if $D_{i}>$ $\left(\frac{3 \ln \Delta}{2}\right)^{i-i_{0}} \cdot \Delta^{i_{0}}$. Now, notice that given $D_{i}, \mathbf{E}\left[D_{i+1}\right]=\frac{\Delta}{k-1} D_{i} \leq D_{i} \ln \Delta$. Thus, by a Chernoff bound, for every $i \geq i_{0}$,

$\operatorname{Pr}[$ failure in level $i+1 \mid$ not failure in level $i] \leq \exp \left(-\frac{\ln \Delta}{16} \cdot\left(\frac{3 \ln \Delta}{2}\right)^{i-i_{0}} \Delta^{i_{0}}\right)$.

Now, since $\operatorname{Pr}$ [ failure in level $i+1] \leq \operatorname{Pr}[$ failure in level $i+1 \mid$ not failure in level $i]+$ $\operatorname{Pr}[$ failure in level $i]$ and since $\operatorname{Pr}\left[\right.$ failure in level $\left.i_{0}\right]=0$, we have by induction that

$$
\begin{aligned}
\operatorname{Pr}[\text { failure in level } \ell] & \leq \sum_{i=i_{0}+1}^{\ell} \operatorname{Pr}[\text { failure in level } i \mid \text { not failure in level } i-1] \\
& \leq 2 \exp \left(\frac{-\Delta^{i_{0}}}{16}\right) \\
& \leq \exp \left(-\Delta^{\varepsilon \ell / 10}\right) .
\end{aligned}
$$

Notice that the event of failure in level $\ell$ includes the event that $D_{\ell} \geq \Delta^{\varepsilon \ell / 8}$ so we are done. $\mathrm{Q}$

Acknowledgments. The authors would like to thank Fabio Martinelli, Elchanan Mossel, Alistair Sinclair, Allan Sly and Prasad Tetali for helpful discussions on the topic.

\section{REFERENCES}

[1] D. Achlioptas and M. Molloy. Analysis of a List-Coloring Algorithm on a Random Graph, in 38th Annual IEEE Symposium on Foundations of Computer Science (FOCS), 204-212, 1997.

[2] D. Aldous. Random walks on finite groups and rapidly mixing Markov chains. Séminaire de Probabilites XVII, Lecture Notes in Mathematics, 986:243-297, Springer Verlag, Berlin, 1983.

[3] N. Bhatnagar, J. Vera and E. Vigoda. Reconstruction for Colorings on Trees. Preprint, 2007. Available from arXiv at: http://arxiv.org/abs/0711.3664v1

[4] N. Berger, C. Kenyon, E. Mossel, and Y. Peres. Glauber Dynamics on Trees and Hyperbolic Graphs, Probab. Theory Related Fields, 131(3):311-340, 2005.

[5] C. Borgs, J. T. Chayes, E. Mossel, and S. Roch. The Kesten-Stigum Reconstruction Bound Is Tight for Roughly Symmetric Binary Channels, in 47th Annual IEEE Symposium on Foundations of Computer Science (FOCS), 518-530, 2006.

[6] P. M. Bleher, J. Ruiz, and V. A. Zagrebnov. On the purity of the limiting Gibbs state for the Ising model on the Bethe lattice, J. Statist. Phys., 79(1-2):473-482, 1995.

[7] G. Brightwell and P. Winkler. Random Colorings of a Cayley Tree, Contemporary Combinatorics, B. Bollobas ed., Bolyai Society Mathematical Studies, 10:247-276, 2002.

[8] G. Brightwell and P. Winkler. A second threshold for the hard-core model on a Bethe lattice, Random Struct. Algorithms, 24(3):303-314, 2004.

[9] C. Daskalakis, E. Mossel and S. Roch. Optimal Phylogenetic Reconstruction, in Proceedings of the 38th Annual ACM Symposium on Theory of Computing (STOC), 159-168, 2006.

[10] P. Diaconis and L. Saloff-Coste. Logarithmic Sobolev inequalities for finite Markov chains, Annals of Applied Probability, 6(3):695-750, 1996. 
[11] D. Dubhashi, D. Ranjan. Balls and bins: a study in negative dependence, Random Struct. Algorithms, 13(2):99-124, 1998.

[12] W. Evans, C. Kenyon, Y. Peres, and L. J. Schulman. Broadcasting on trees and the Ising model, Annals of Applied Probability, 10(2):410-433, 2000.

[13] A. Frieze and R. Kannan. Log-Sobolev inequalities and sampling from log-concave distributions, Annals of Applied Probability, 9(1):14-26, 1999.

[14] A. Frieze and E. Vigoda. A survey on the use of Markov chains to randomly sample colorings. Combinatorics, Complexity and Chance, G. Grimmett and C. McDiarmid eds., Oxford University Press, 2007.

[15] H. O. Georgii. Gibbs Measures and Phase Transitions, de Gruyter Studies in Mathematics, vol. 9, 1988.

[16] A. Gerschenfeld and A. Montanari. Reconstruction for models on random graphs, in 48th Annual IEEE Symposium on Foundations of Computer Science (FOCS), 197-204, 2007.

[17] L. Goldberg, M. Jerrum, and M. Karpinski. The mixing time of Glauber dynamics for colouring regular trees. Preprint, 2008. Available from arXiv at: http://arxiv.org/abs/0806.0921

[18] T. P. Hayes, J. Vera, and E. Vigoda. Randomly coloring planar graphs with fewer colors than the maximum degree, in Proceedings of the 39th Annual ACM Symposium on Theory of Computing (STOC), 450-458, 2007.

[19] J. Jonasson. Uniqueness of uniform random colorings of regular trees, Statistics and Probability Letters, 57:243-248, 2002.

[20] F. Krzakala, A. Montanari, F. Ricci-Tersenghi, G. Semerjian and L. Zdeborová. Gibbs States and the Set of Solutions of Random Constraint Satisfaction Problems, Proc. Natl. Acad. Sci. USA, 104(25):10318-10323, 2007.

[21] B. Lucier, M. Molloy, and Y. Peres. The Glauber dynamics for colourings of bounded degree trees. To appear in Proceedings of the 13th Intl. Workshop on Randomization and Computation (RANDOM), 2009.

[22] J. Martin. Reconstruction thresholds on regular trees, in Discrete Random Walks, DRW'03, C. Banderier and C. Krattenthaler eds., Discrete Mathematics and Theoretical Computer Science Proceedings, 191-204, 2003.

[23] F. Martinelli, A. Sinclair, and D. Weitz. Glauber dynamics on trees: Boundary conditions and mixing time, Communications in Mathematical Physics, 250(2):301-334, 2004.

[24] F. Martinelli, A. Sinclair, and D. Weitz. Fast mixing for independent sets, colorings and other models on trees, Random Struct. Algorithms, 31(2):134-172, 2007.

[25] A. Montanari and M. Mézard. Reconstruction on trees and spin glass transition, J. Stat. Phys., 124:1317-1350, 2006

[26] A. Montanari, R. Restrepo and P. Tetali. Reconstruction and Clustering in Random Constraint Satisfaction Problems, Preprint, 2009. Available on arXiv at: http://arxiv.org/abs/0904.2751

[27] R. Montenegro and P. Tetali. Mathematical Aspects of Mixing Times in Markov Chains, Foundations and Trends in Theoretical Computer Science, 1(3), 2005.

[28] E. Mossel. Phase transitions in phylogeny, Trans. Amer. Math. Soc., 356(6):2379-2404, 2004.

[29] E. Mossel. Survey: Information Flow on Trees, Graphs, Morphisms and Statistical Physics, DIMACS series in discrete mathematics and theoretical computer science, J. Nešetřil and P. Winkler, eds., 155-170, 2004.

[30] E. Mossel and Y. Peres. Information flow on trees, Annals of Applied Probability, 13(3):817-844, 2003.

[31] D. Weitz. Mixing in Time and Space for Discrete Spin Systems, Ph.D. thesis, UC Berkeley, 2004.

[32] L. Saloff-Coste. Lectures on finite Markov chains, Lectures on probability theory and statistics (Saint-Flour, 1996), Lecture Notes in Mathematics, 1665:301-412, Springer Verlag, Berlin, 1997.

[33] G. Semerjian. On the Freezing of Variables in Random Constraint Satisfaction Problems, Journal of Statistical Physics, 130(2):251-294, 2008.

[34] A. Sly. Reconstruction of Random Colourings. Preprint, 2008. To appear in Communications in Mathematical Physics, Available on arXiv at: http://front.math.ucdavis.edu/0802.3487

[35] L. Zdeborová and F. Krzakala. Phase Transitions in the Coloring of Random Graphs, Phys. Rev. E, 76, 031131, 2007. 